\title{
Personal determinants of professional development of the future specialist
}

\author{
Tatyana Savchenko \\ Omsk Academy of the Humanities, Omsk, Russia
}

\begin{abstract}
The article highlights the issues of identifying personal determinations of the development of future specialists, and in particular, physical education teachers in their professional training, substantiates and empirically tests the dependence of the professional development of a future physical education teacher on his personal qualities. The author analyzes the features of the professional development of future physical education specialists in the article. Personal and professional development is viewed as an integral self-developing system based on the activity-based transformation of the personality of itself. The author identifies personal determinants that affect the professional development of a future specialist, such as professional orientation, consisting of an interest in the teaching profession and the desire to master knowledge and skills; a pedagogical vocation, which grows out of the awareness of the abilities for the profession of a physical education teacher. The author presents the results of this study, presents conclusions about the effectiveness of the experiment.
\end{abstract}

\section{Introduction}

An important issue of modern vocational education is to identify the determinations of the development of future specialists and, in particular, physical education teachers, in their professional training. The relevance of this issue is due to the trends of modern world development, the increase in competition and profound structural changes in the labour market, which fully applies to the physical education teacher.

In this regard, it is of interest to study this problem to identify the dependence of the professional development of future specialists on their personal characteristics. The main idea of our study is to identify the personal determinations of the development of future physical education specialists in their professional education.

The development of the professional characteristics of a teacher's personality is a continuous process that requires regular work on self-development, professional development and the accumulation of experience in social and creative activities. This will allow the teacher to be at the centre of modern changes in the professional environment, and, if the new requirements are successfully met, to be in demand throughout his career, to withstand high competition in the labour market. In addition, such a teacher has a steady demand for the provision of services, the conditions for achieving full-fledged professional development are observed. 


\section{Methods and types of the Earth's remote sensing}

The development of the personality of a competitive physical education teacher, who is in demand by society, begins at a very early age and then continues through a career. Qualitative characteristics of teacher training, readiness to apply social roles and professional responsibilities are analyzed by a list of criteria that are specific to a particular pedagogical field. The main criteria for the formation of a professional model of a teacher can be called his personal determinants.

In our view, the professional and personal development of a future specialist, the selfdetermination of his personality and its self-actualization depend on a certain mobilization of a person's inner powers, on the growth of his internal reserves and their harmonious and rational use. It is conditioned by the search for incentives and motives for the activity orientation of the personality and its concentration on goals. This was repeatedly mentioned by famous scientists, such as: A.A. Bodalev, A.N. Leontiev and others.

When revealing the question of the determinants of professional development of a future physical education teacher, it makes sense to rely on the studies of S.L. Rubinstein, who, taking the general principle of determinism as a basis, explains all the actions of external factors by their interpretation through the personality, as an integral system of external factors. Mental, as S.L. Rubinstein believed, despite the existing specifics, is not something special, which is opposed, by its very nature, to the phenomena of the material world. Both material and mental are closely related and form an integral phenomenon, the so-called being, in which the psyche representing the highest level of this being and distinguished by a specific nature, acts as "not only as conditioned but also as a conditioning", a phenomenon that determines, regulates, directs human activity [1].

According to many authors, personality is not a bundle of individual components, personality traits, personality in their view is an integral, stable system of many interrelated and interacting characteristics.

As for the professionally important personal qualities, we associate with them those individual characteristics of the subject of activity that affect its success and the effectiveness of mastering the activity. Abilities can also be attributed to professionally important personal qualities. Abilities determine the essential pedagogical component of the continuous accumulation and manifestation of the potential of the individual (the unity of the possible and the accomplished, potential and actual), which contributes to the development of the personality, the expansion of its ties with society, other people, with the world around it and with oneself under the influence of many factors: hereditary, psychological, social and pedagogical. The process of personal development is provided by "upbringing", education, self-education, pedagogical support, organized socialization [2].

In the structure of the teacher's personality, it is possible to distinguish such constituent elements as social and vocational orientation, motives of professional and pedagogical activity, professional and pedagogical interests, pedagogical duty and responsibility, pedagogical vocation.

Regarding the skill of the physical education teacher, which creates the identity of his communication with students, the nature of interaction with them, then it is largely determined by those qualities that contribute to the mastery of various skills, determine the speed and success of their acquisition. These might include such important characteristics as communicative, volitional, moral, intellectual and psychomotor. A physical education teacher should be distinguished by a full set of moral characteristics: humanism, politeness, honestly, exactingness, optimism and other moral traits, because the moral education of students is primarily based on the personal example of the teacher, and not on his external influence. A physical education teacher is obliged to constantly take care of himself, keep and maintain the physical condition of the body, make high moral demands on himself. 
The prerequisite for the success of a teacher's activity, and in particular a physical education teacher, is the development of his volition. The key volitional characteristics of a teacher are considered: his commitment, courage, determination, restraint and self-control. All these characteristics are interrelated and interdependent. The fact that a teacher possesses such qualities in the psychomotor sphere, which affect the accuracy of the performance of techniques when they are shown and such qualities of the perceptual sphere, which are responsible for the accuracy of perception, movements of the sportsman, the speed of perception and assessment of the situation, is a consequence of the requirements imposed on the activity of a physical education teacher. For a physical education teacher, it is important to have good physical training and good physical shape, since he himself has to show exercises that require him to be physically strong, flexible, agile, quick to react, and such a physical shape must be kept and maintained at the proper level throughout all teaching activity.

The professional activity of a teacher is an integral part of the pedagogical system, which is characterized by a purposeful influence on the students using psychologically sound pedagogical means. The implementation of the pedagogical system is expressed in the structure of the pedagogical activity of the physical education teacher himself, as well as through the prism of a number of psychological characteristics [2].

The main components of the structure of pedagogical activity are distinguished on the basis of the functions that he performs. The priority of the teacher's activity includes such functional components as gnostic, design, constructive, organizational, communicative [2].

The professional activity of the teacher is to perform the functions of planning, organization and control. The structural elements of the teacher's activity are highlighted taking into account the results of the analysis of the stages presented.

Thus, the physical education teacher is called upon to solve the problems of the all-round development of the personality of the students. For the successful implementation of the education process, a teacher needs to understand that the activity of a physical education teacher largely depends on the characteristics of the organization of the pedagogical process, the prevailing social conditions, the formed personal potential of the teacher and on his ability to meet today's changes in education. At the same time, his professional productivity is manifested through his psychological component, which includes: his interests, value orientations, motives and goals of the activity, the level of the intellectual development of the teacher. A modern teacher must have the ability to create the necessary atmosphere of creative participation of students, through which the interaction of students with the teacher is realized. The structural components of the teacher's success may include procedural, effective and personal aspects if by the effective component we mean the education and training of students, and by the personal component we mean the characteristics of the personality of a physical education teacher [1]. At the same time, the development of the personality of a physical education teacher is considered as his self-realization, an incentive to creativity, the need to design his own professional level at various stages of his life [3].

A modern physical education teacher should be a full-fledged person, morally, intellectually and physically ready to actively take up his job, to carry out work that is meaningful for himself and for society in difficult conditions of increasing competition. But it should be noted that the process of training specialists and their competence leaves much to be desired. The way out of this situation is seen in the actualization of the personality of the individual, when, along with the assimilation of specialized knowledge and the achievement of the proper level of development of professional abilities, the acquisition of social values, familiarization with culture become a necessary condition for the employee's personality to meet the continuously increasing requirements of occupational work [1].

The peculiarity and uniqueness of pedagogical activity and, in general, of the entire teaching profession is manifested in the peculiarities of its understanding and 
implementation. It belongs to a group of professions in which another person is the subject of influence. But its distinctive feature and its uniqueness is that representatives of this profession differ from others in their way of thinking, increased responsibility for others, and a strong sense of duty. In this regard, the teaching profession can be distinguished into an independent group of professions of the "person-person" type. Its main characteristic is that it belongs to both the class of transformative and the class of managing occupations at the same time. The task of the teacher in the educational process is not only to change and transform the student's personality but to manage the entire process of his intellectual, emotional, physical development and the process of the spiritual transformation of his personality. The content of the activity of the teaching profession, as well as the activity of representatives of professions of the "person-person" type, constitutes a relationship with another person, but the goals and values of these relationships in the teaching profession are different and are related to understanding the student, shaping his aspirations and directing them towards achieving social goals.

The appeal of modern pedagogical education to the personality of the future teacher as a subject of communication, knowledge and social creativity increases the role of the student in mastering the teaching profession. He must clearly understand the purpose and objectives of his activity, clearly see the ways of professional development [2].

One of the important methodological problems of the development of pedagogical activity is the selection and understanding of the subject and object of activity.

S.L. Rubinstein emphasized in his studies that consciousness is irreducible to only one subjectivity, it, in the opinion of S.L. Rubinstein, is the unity of the objective and the subjective, and when an event is realized, the process of including the experiences of the subject into objective subject-matter relationships associated with the subject takes place. In our studies, we consider the concept of "subjective" as one of the characteristics of consciousness, and "subjective" - as inherent in the subject.

According to the researcher A.K. Markova, important signs of human subjectivity are his awareness of the structure of his activity. This includes an understanding of the qualities of one's personality, the content of the stages of life path, goal-setting, initiative, planning of one's activities, etc. Subjectivity can be characterized by the intense involvement of a person in the activities, the presence of self-control, self-correction, and possession of self-regulation techniques.

The formation of the subject, according to S.L. Rubinstein, goes through a number of stages: firstly, a person by his being strengthens the being of another, his individual and personal characteristics; secondly, the subject refers to the other as to the fact of the formation of his nature; thirdly, it recognizes the right of others to self-determination and freedom. At the same time, for the subject, another person appears as a subject capable of changing the world and himself. The orientation of the personality characterizes the person as a whole, i.e. what he has in his experience and what constitutes the area of application of his knowledge, abilities, skills. The cognitive structure shows human capabilities, the constituent components of which are: attention, thinking, memory, perception, sensation and imagination. The distribution of attention is a qualitative characteristic of the ability to simultaneously concentrate consciousness on several phenomena in order to see the overall picture of the situation.

In practice, apperception can play an important role, and this is the experience, knowledge, abilities, skills, attitudes of a person.

Professional training is a process that, in its multifaceted structure, includes aspects of a pedagogical orientation, which is an integral indicator of a teacher's personality, as well as an object of his self-development in the professional sphere [5].

The gnostic component consists of actions to research and accumulate information about the goals of the entire system, means of achievement, the level of development of participants 
in the pedagogical process, age characteristics, individual psychological characteristics, ways of interacting with students, as well as the results of their activities, taking into account positive and negative qualities.

The projective component consists of actions related to the development and planning of goals, planning the activities of schoolchildren for a long period, planning the teacher's activities [5].

The constructive component consists of actions related to the selection and construction of teaching and educational process, the choice of teaching methods, modelling the behaviour of students during the implementation of various forms of work, modelling behaviour in future professional activities.

The communicative component consists of actions that make it possible to establish pedagogically expedient relationships in the "teacher-student" system, motivate the activities of the subjects of the educational process, and penetrate the imaginative world of the subjects of the learning process [4].

The organizational component consists of actions that help the implementation of the pedagogical scenario, the organization and functioning of actions in the "teacher-student" system, the introduction of pre-developed models of behaviour and algorithms for the activities of the subjects of the educational process [5].

The educational process can be considered as a system of interaction between a teacher and a student.

The professional orientation of a teacher can be characterized as a rather complex concept, which includes, first of all, interest in this profession, and the associated desire to engage in his own activities, to have a positive attitude towards interacting with his students, their parents, the desire to master knowledge, skills, and improve his professional achievements.

The pedagogical vocation shows love for people, for children, a desire to do good, to give away one's knowledge. This characteristic is formed in the process of accumulating knowledge and experience in pedagogical activities, is fundamental in the process of selfimprovement of the teacher.

The skill of a physical education teacher is determined by the abilities for professional activity, among which it is possible to distinguish: didactic abilities - the abilities involved in the transfer of educational material, which are associated with the teacher's ability to reconstruct and adapt educational material; academic abilities - the abilities that allow the teacher to master his subject, both in the volume of the required curriculum and much wider; strive to master the latest discoveries in the field of scientific knowledge of his subject; perceptual abilities associated with the subtle perception of the student's personality; verbal and organizational abilities. Authoritarian, communicative and several other abilities that allow you to directly influence the emotional-volitional sphere of schoolchildren, as well as the teacher to reflect on his behaviour. It is necessary for the teacher to show conviction and interest in what he or she said [3].

In the realities of modern life, the attitude towards the professional work of a teacher is significantly changing. The requirements for the level of his personal and professional development are increasing, which provides for a qualitatively different level of formation of the teacher's motivational sphere, his professional and pedagogical thinking, and the ability for constant self-development and self-improvement.

Our study took place on the basis of BPEI Omsk Pedagogical College No. 1. The study involved 4th-year students enrolled in Program 49.02.01. "Physical culture", in the amount of 25 people. When studying the scientific and methodological literature, we identified the following personal qualities on which the future success of the professional development of a physical education teacher depends. We focused on the study of two subject areas, where one of the most important components is the cognitive sphere. This is what allows you to 
gain knowledge and ultimately successfully navigate in a constantly changing environment. Its constituent factor is: attention, thinking, memory, perception, sensation and imagination. The second component in the study of the subject areas is the subject-personal sphere. From the point of view of pedagogy, it is this that acts as the object and subject of the pedagogical process, teaching, upbringing and development. The formation of the personality is influenced by several factors, where social circumstances play a leading role in the formation of the personality.

To solve the set tasks and achieve the goal of the study, both theoretical and empirical methods were used in the work: pedagogical observation was used to study the behaviour of students in the process of their interaction with each other, during training sessions and after school hours. The presented "battery" of psychodiagnostic techniques is aimed at studying the variety of professionally important properties and qualities of a future specialist in the physical sphere. The materials were processed using standard methods of mathematical statistics. For quantitative calculation and the following qualitative data processing, the following values were calculated in the work: \% - percentage; $\bar{X}$ - average; $\sigma$ - standard deviation; $r$ - correlation coefficient.

The pedagogical experiment was conducted in order to reveal the effectiveness of the social-pedagogical training developed by us to identify the personal conditionality of the professional development of a future physical education specialist. Individual and mass forms of work were used in the program. At the end of the pedagogical experiment, the study results were processed and interpreted.

The expert assessment was conducted to assess the personal potential of the professional success of the 4th year students of the BPEI "OPC No. 1".

In our study, the expert assessment of the personal properties and qualities of a future physical education specialist is based on quantitative parameters and evaluation criteria obtained as a result of the analysis of scientific and methodological literature. The essence of the quantitative assessment, which makes it possible to assess the success of a student's professional development, is that qualitative characteristics are highlighted in his activities, each of which is assessed independently of the others (in points). Based on these independent private assessments, the integral indicator of the expert assessment was calculated.

\section{Analysis of the main ERS data sources for the DEM development}

Within the framework of the psychodiagnostic study, the manifestations of two spheres of personality were studied: cognitive and subjective-personal. When diagnosing the cognitive sphere, we found that for a future PE (physical education) specialist, the most characteristic is the subject-effective type of thinking ( $\bar{X}=6.8$ points), an expressed preference or persistent ignorance of one or another style of thinking was not found in most students. The study of students' preferred learning styles indicates a clear advantage of their choice $(50 \%$ of students) of an active learning style.

The main characteristics of the subjective-personal sphere include the following indicators: among students, indicators prevail on the following scales: social ergicity $(\bar{X}=$ 9.7 points), tempo ( $\bar{X}=9.5$ points $)$ and social tempo ( $\bar{X}=9.1$ points $)$, which indicates the orientation of their personality towards the development of social forms of activity, the desire for leadership, characterizes them as sociable people and involved in social activities. The tempo $(\bar{X}=9.5)$ indicates the speed of their performance of individual operations, the speed of motor and movement acts when performing substantive activities. Increased indicators on 
the scale "social tempo" ( $\bar{X}=9.17)$ are associated with the acceleration of the rhythm of modern life, an increase in the speed of communication between young people.

The overwhelming majority of students $(10 \%)$ have underestimated self-criticism, while in aggregate $80 \%$ of the respondents found an adequate $(45 \%)$ or inflated (35\%) level of selfesteem. The majority of students $(80 \%)$ show a moderate risk appetite, while only $5 \%$ of respondents are characterized by a high risk appetite and $15 \%$ of respondents - a low one.

The results of the expert assessment allow us to draw the following conclusion: the most developed quality among students is "focus on achieving results" ( $\bar{X}=3.45$ points), the lowest value is assigned to such quality as "ability to reflect" ( $\bar{X}=3.07$ points). One of the most highly rated indicators was "the desire for creative search" ( $\bar{X}==3.31$ points). Low enough values were assigned to the indicator "communicativeness" ( $\bar{X}==3.16$ points), a high result of the expert assessment is on the indicator "ability to self-organization" ( $\bar{X}==$ 3.25 points). The integral expert indicator for the group was ( $\bar{X}==3.24$ points).

Based on the results obtained in the course of studying the cognitive and subject-personal spheres, we have developed a socio-pedagogical training "I am a physical education teacher" for the development of personal qualities of future physical education teachers. According to the results of the socio-pedagogical training and the method of pedagogical observation, the following can be stated. During the implementation of the business game, the qualities shown in the group discussion were assessed: the highest value according to the results of the assessment was revealed by the indicator "activity" ( $\bar{X}=3.37$ points). We believe that a sufficiently high indicator confirms the previously obtained results about the readiness of students to take an active position in the learning process. It was also the first time that students took part in such a form of work, which, of course, could motivate them to be active.

We estimate the value on the scale "readiness to work in a team" ( $\bar{X}=3.25$ points) very positively for the formation of a physical education specialist. The teacher's job often involves making collective decisions, which is associated with the skills of cooperation and mutual understanding, therefore, this indicator is very important from the point of view of the effectiveness of future professional activities.

Students' initiative is characterized by an average value ( $\bar{X}=3.0$ points), but it should be noted that some students willingly showed initiative, expressing their position on the issues of their future colleagues, showed a desire to regulate the forms of group work.

The lowest values were obtained in terms of indicators "constructiveness" and "decisionmaking" ( $\bar{X}=2.87$ points). We attribute this to the fact that for students the situation of collective discussion of upcoming reports was limited by time, characterized by novelty and lack of experience of such work in the educational process. As for the direct decision-making, here, in our opinion, the main factor was the unwillingness of students to take responsibility for the result of their colleague's speech.

As a result, the integral indicator of the assessment of the group discussion was $(\bar{X}=$ 3.07 points). Taking into account the above discussion of the results, we believe that this value is acceptable for this stage of professional training, but requires a qualitative improvement through the introduction of active forms of education.

When assessing the presentations made by the participants in the socio-psychological experiment, the following results were obtained: the lowest indicators were identified according to the criterion "content of the report" ( $\bar{X}=2.46$ points), the students showed the highest indicator according to the criterion "ability to hold on" ( $\bar{X}=3.59$ points), the average scope according to the criterion "persuasiveness of arguments" was ( $\bar{X}=3.33$ points). 


\section{Conclusions}

As a result of identifying personal qualities that are more promising in terms of professional development and further growth, it was determined: such variables as active experimentation and the manifestation of students' activity $(r=0.68)$ are objectively dependent, a reliable relationship was revealed with indicators of students' social ergicity and readiness to work in a team $(r=0.9)$, a low level of self-criticism and social emotionality determined the presence of a negative correlation with the same indicators - readiness to work in a team $(r=-0.83$ and $\mathrm{r}=-0.69$ respectively). A positive relationship between inflated self-esteem and student initiative was found $(\mathrm{r}=0.79) /($ Fig. 1)

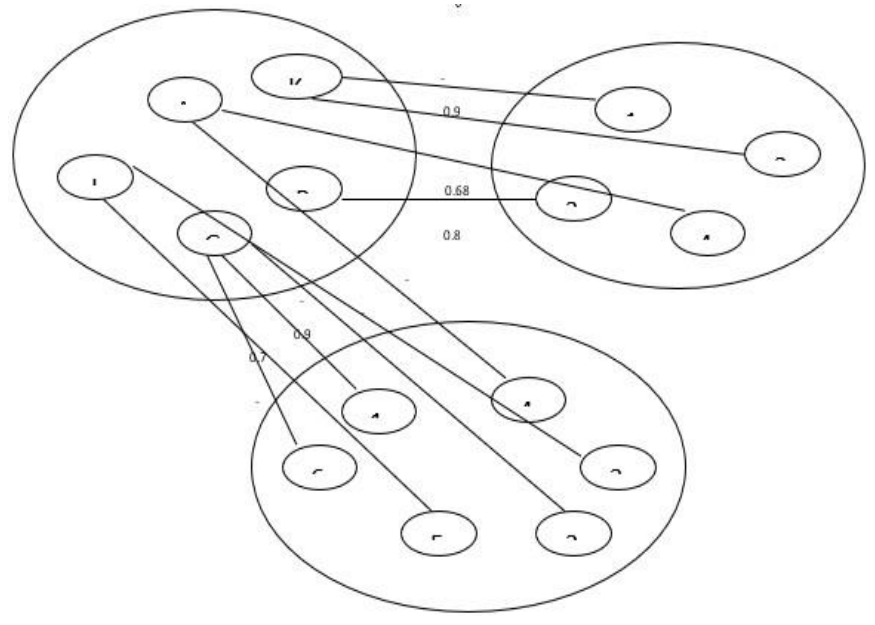

Fig. 1. Correlation between personality spheres.

Symbols: A - activity, I - initiative, $\mathrm{G}$ - readiness to work in a team, $\mathrm{K}$ - constructiveness, $\mathrm{P}$ - decision-making; $1 \mathrm{~K}$ - level of mental abilities, $2 \mathrm{~K}$ - verbal-logical type of thinking, 3 analytical style of thinking, $4 \mathrm{~K}$ - active experimentation; $1 \mathrm{C}$ - social ergicity, $2 \mathrm{C}$ - selfcriticism, 3C - motivation, 4C - level of aspiration, 5C - self-esteem, 6C - social emotionality

The results of psychodiagnostics, expert assessment, data of socio-pedagogical training and pedagogical observation allow us to reflect in the thesis form the requirements for the process of professional training of physical education teachers in the specifics of their occupation. We believe that there is a need to develop and introduce into the educational process the professional training of a physical education teacher:

- special educational courses (elective courses, electives) to orient students to active educational, practical and social activities;

- innovative forms of work that allow you to model quasi-professional activities of students;

- diagnostic methods to assess the results of the process of professional development, determine the growth points of the student in the perspective of future specialization (observation diaries, expert protocols, ranking scales).

\section{References}

1. S. P. Akutina, Sociosphere, 1, 54 (2015)

2. A. B. Muller, Physical culture, 424 (2018)

3. V. F. Strelchenko, L. N. Koval, E. N. Alekseeva, Research publications, 9, 33 (2014) 
4. Z. M. Bagautdinov, World of science, culture, education, 1, 27 (2015)

5. I. N. Boyko, Theoretical and applied aspects of modern science, 8(4), 80 (2015)

6. E. A. Magazeva, Omsk Scientific Bulletin, 1 (125), 140 (2014)

7. S. A. Khazova Development of competitiveness of specialists in the process of professional training: Monograph, 24, 1 (2011)

8. L. M. Manuylova, T. V. Savchenko, Omsk Scientific Bulletin, 6(102), 221 (2011)

9. A. N, Kapustin, Prospects of science and education, 1 (2013) http://pnojournal.wordpress. com 